\title{
Classical large solution for the viscous compressible fluids with initial vacuum in 1D
}

\author{
Zhilei Liang ${ }^{1}$ and Yunzhao Lu*
}

*Correspondence: 59604292@qq.com

${ }^{2}$ School of International Trade and Economics, University of

International Business and Economics, Beijing, 100029, P.R. China

Full list of author information is available at the end of the article

\begin{abstract}
This paper is concerned with the Cauchy problem for a compressible viscous fluid in one-dimensional (1D) space. By means of the weighted initial density, we obtain the global-in-time existence of a unique classical solution with large initial data. The initial density can be compactly supported or decays to zero not too slowly at infinity.
\end{abstract}

Keywords: compressible Navier-Stokes; global classical solution; vacuum; Cauchy problem

\section{Introduction}

The viscous isentropic compressible fluid in one-dimensional (1D) space is governed by the compressible Navier-Stokes equations,

$$
\left\{\begin{array}{l}
\rho_{t}+(\rho u)_{x}=0, \\
(\rho u)_{t}+\left(\rho u^{2}\right)_{x}+P_{x}=\mu u_{x x}, \quad x \in \mathbb{R}, t>0,
\end{array}\right.
$$

where the unknown functions $\rho(x, t), u(x, t)$, and $P=K \rho^{\gamma}(K>0, \gamma>1)$ are the density, velocity, and pressure, respectively. The viscosity coefficient $\mu>0$ is a given constant.

We are interested in the existence of a classical solution to (1.1) with the far field behavior

$$
(\rho, u) \rightarrow(0,0) \quad \text { as }|x| \rightarrow \infty, t \geq 0,
$$

and initial data

$$
(\rho, u)(x, 0)=\left(\rho_{0} \geq 0, u_{0}\right)(x), \quad x \in \mathbb{R}
$$

We first review briefly the well-posedness of solutions for (1.1). Non-vacuum small perturbations around a constant have shown that the solutions are classical and globally defined in time if the initial data are sufficiently regular; see [1-4]. If the initial vacuum is allowed, the global weak solutions were first obtained by Lions [5] for the isentropic fluids (see also Feireisl [6]) for large initial data. Later, some regularity information was obtained in [7, 8]. When it comes to the strong/classical solutions, Kim et al. [9-11] proved the local existence and uniqueness for both bounded and unbounded domains $\Omega \subseteq \mathbb{R}^{3}$ (this also holds for bounded domains in $\mathbb{R}$ or $\mathbb{R}^{2}$ ). Based on the a priori estimates developed [9-11], Ding et al. considered the initial-boundary-value (IBV) problem in 1D space, and making use of 1D properties, they [12] obtained the global existence of classical solution with

(c) 2015 Liang and Lu. This article is distributed under the terms of the Creative Commons Attribution 4.0 International License (http://creativecommons.org/licenses/by/4.0/), which permits unrestricted use, distribution, and reproduction in any medium, provided you give appropriate credit to the original author(s) and the source, provide a link to the Creative Commons license, and indicate if changes were made. 
large initial data and vacuum. Similar results was obtained for the annular (or exterior) domain in $\mathbb{R}^{2}$ and $\mathbb{R}^{3}$ under the spherically symmetric assumption; see [13, 14]. Huang et al. [15] showed that for the 3D Cauchy problem there exists a unique classical solution in the case when the initial total energy is small, but there possible are large oscillations and a vacuum.

However, the existence of a classical solution for the Cauchy problem in 1D (or 2D) is another issue. In particular, the argument in [9-11] could not be directly applied if the domain $(N=1,2)$ becomes unbounded since the $L^{p}$-norm $(p \geq 2)$ of the velocity cannot be controlled just in terms of the $L^{2}$-norm of the gradient of it. Li and Liang [16] obtained the local existence and uniqueness of strong and classical solutions to the 2D Cauchy problem with the vacuum as a far field density. The key idea in [16] is to control $\|\rho u\|_{L^{p}}$, instead of $\|u\|_{L^{p}}$, in terms of $\left\|\rho^{1 / 2} u\right\|_{L^{2}}$ and $\|\nabla u\|_{L^{2}}$ by introducing a weight to the initial density. Recently, Li and Xin [17] showed that the classical solution for 2D Cauchy problem exists globally in time in the case of small initial energy; furthermore, some large-time decay rates of solutions are first presented.

In this paper, for $1 \leq r \leq \infty$ and integer $k \geq 0$, we adopt the simplified notations for the standard homogeneous and inhomogeneous Sobolev spaces

$$
L^{r}=L^{r}(\mathbb{R}), \quad D^{k}=\left\{f \in L_{\mathrm{loc}}^{1}(\mathbb{R}):\left\|\partial_{x}^{k} f\right\|_{L^{2}(\mathbb{R})}<\infty\right\}, \quad H^{k}=L^{2} \cap D^{k} .
$$

The concern of this paper is the existence, uniqueness, and large-time behavior of classical large solutions to the Cauchy problem (1.1)-(1.3), with the vacuum as a far field state, even for the compactly supported density.

\section{Theorem 1.1 Define}

$$
\bar{x}=\left(e+x^{2}\right)^{1 / 2} \ln \left(e+x^{2}\right) .
$$

Assume that the initial functions $\left(\rho_{0}, u_{0}\right)$ satisfy for $a \in[3, p)$

$$
\begin{aligned}
& \bar{x}^{a} \rho_{0} \in L^{1} \cap H^{1}, \quad \rho_{0}^{1 / 2} u_{0} \in L^{2}, \quad u_{0} \in D^{1} \cap D^{2}, \\
& \rho_{0 x x}, P_{0 x x} \in L^{2}, \quad \rho_{0}^{1 / p} u_{0} \in L^{p}, \quad u_{0}^{p / 2} \in D^{1},
\end{aligned}
$$

and the following compatibility condition:

$$
-\mu u_{0 x x}+P_{0 x}=\rho_{0}^{1 / 2} g, \quad \text { for some } g \in L^{2} \text {. }
$$

Then the Cauchy problem (1.1)-(1.3) admits a unique classical solution $(\rho, u)$, which satisfies for any $T \in(0, \infty)$

$$
\left\{\begin{array}{l}
\rho \in C\left([0, T] ; L^{1} \cap H^{2}\right), \quad P \in L^{\infty}\left(0, T ; H^{2}\right), \\
\bar{x}^{a} \rho \in L^{\infty}\left(0, T ; L^{1} \cap H^{1}\right), \quad \rho u \in L^{\infty}\left(0, T ; L^{1} \cap H^{1}\right), \\
u_{x} \in L^{\infty}\left(0, T ; L^{\infty} \cap H^{1}\right), \\
\rho^{1 / p} u \in L^{\infty}\left(0, T ; L^{2}\right), \quad \partial_{x}\left(u^{p / 2}\right) \in L^{2}\left(0, T ; L^{2}\right), \\
\bar{x}^{-1} u \in L^{\infty}\left(0, T ; L^{2} \cap L^{\infty}\right), \quad \bar{x}^{-2 / p} u \in L^{p}\left(0, T ; L^{p} \cap L^{\infty}\right), \\
\rho^{1 / 2} \dot{u} \in L^{\infty}\left(0, T ; L^{2}\right), \quad \dot{u}_{x} \in L^{2}\left(0, T ; L^{2}\right), \\
t^{1 / 2} u_{x x x}, t^{1 / 2} \dot{u}_{x}, t \dot{u}_{x x} \in L^{\infty}\left(0, T ; L^{2}\right), \\
t \rho^{1 / 2} \ddot{u} \in L^{\infty}\left(0, T ; L^{2}\right), \quad t \ddot{u}_{x} \in L^{2}\left(0, T ; L^{2}\right),
\end{array}\right.
$$


where

$$
\dot{u}=u_{t}+u u_{x} \quad \text { and } \quad \ddot{u}=\dot{u}_{t}+u \dot{u}_{x}
$$

denote the material derivatives of $u$ and $\dot{u}$, respectively.

Remark 1.1 We can check that the solution $(\rho, u)$ in Theorem 1.1 satisfying $(1.7)$ is in fact a classical one.

Remark 1.2 We mention [18] by Xin, where the author shows the solution $(\rho, u) \in$ $C\left([0, T] ; H^{3}(\mathbb{R})\right)$ must occur with blowup phenomena in the case that the non-trivial initial density has a compact support.

Remark 1.3 The hypothesis $P_{0 x x} \in L^{2}$ in (1.5) can be removed when $\gamma \geq 2$.

Following some ideas developed by Li and Xin [17] for the multi-dimensional case, $N=$ 2,3 , we give the following $L^{r}$-norm $(r>2)$ estimate on the pressure.

Corollary 1.1 Let $(\rho, u)$ be one of the solutions described in Theorem 1.1. Then it satisfies

$$
\int_{0}^{+\infty}\|P(\cdot, t)\|_{L^{r}}^{r} d t \leq C, \quad \forall r \in[2+1 / \gamma,+\infty)
$$

This work was initially motivated by Ding et al. [12], where the authors considered the global existence of classical solution for the 1D IBV problem. In contrast with [12], the problem under our consideration lies in an unbounded domain, and thus the $L^{p}$-norm of the velocity $u$ could not be dominated just in terms of the $L^{2}$-norm of the gradient of it. In this connection, we follow some ideas in [16] and introduce a weight function to the initial density. However, the usage of a Sobolev embedding inequality in $\mathbb{R}$ is very different from $\mathbb{R}^{2}$. For this, we use some special properties in one-dimensional space (see (2.18)). With these preparations, as well as some ideas in $[1,9,12,17,19]$, we establish the global existence and uniqueness of the classical solution to the Cauchy problem (1.1)-(1.3).

In the rest of this paper, we first derive some global a priori estimates in Sections 2 and 3. This is necessary when we extend the local solution to all positive time in Section 4, and thus complete Theorem 1.1. Finally, Corollary 1.1 is proven in Section 5.

\section{A priori estimates (I)}

We suppose that $(\rho, u)$ is a classical solution to (1.1)-(1.3) over the interval $[0, T]$ with $T \in$ $(0, \infty)$. For simplicity reasons, we may choose $\left\|\rho_{0}\right\|_{L^{1}}=1$, which implies

$$
\int_{-N_{0}}^{N_{0}} \rho_{0}(x) d x \geq \frac{1}{2} \int_{\mathbb{R}} \rho_{0}(x) d x=\frac{1}{2}
$$

for some large number $N_{0}$.

First of all, multiplying $(1.1)_{2}$ by $u$ and integrating by parts, we get

\section{Lemma 2.1 We have}

$$
\sup _{0 \leq t \leq T}\left(\left\|\rho^{1 / 2} u\right\|_{L^{2}}+\|P\|_{L^{1}}\right)+\mu \int_{0}^{T}\left\|u_{x}\right\|_{L^{2}}^{2} d t \leq C,
$$


where as below $C$ denotes a generic constant depending on $\mu, \gamma, K$, a, and the initial data; particularly, the expression $C(\alpha)$ emphasizes that $C$ depends on $\alpha$.

The next lemma derives the bound on the density $\rho(x, t)$ from above.

Lemma 2.2 We have

$$
\rho(x, t) \leq C, \quad(x, t) \in \mathbb{R} \times[0,+\infty) .
$$

Proof The proof is almost exactly the same as that in [19], Lemma 2.3. Here we state the details for completeness. By $(1.1)_{1}$, one has for $t \in[0, \infty)$

$$
\|\rho(t)\|_{L^{1}}=\left\|\rho_{0}\right\|_{L^{1}}=1
$$

Put

$$
\xi(x, t)=\int_{-\infty}^{x} \rho u(y, t) d y
$$

We express $(1.1)_{2}$ in the form

$$
\xi_{x t}+\left(\rho u^{2}\right)_{x}=\left(\mu u_{x}-P\right)_{x}
$$

which, along with $(1.1)_{1}$, provides us after integration in variable $x$ with

$$
\xi_{t}+\rho u^{2}=\mu u_{x}-P=-\mu \frac{1}{\rho}\left(\rho_{t}+u \rho_{x}\right)-P .
$$

Hence

$$
\begin{aligned}
\frac{d}{d t} \xi(X(t, x), t) & =-\mu \frac{d}{d t} \ln \rho(X(t, x), t)-P(X(t, x), t) \\
& \leq-\mu \frac{d}{d t} \ln \rho(X(t, x), t),
\end{aligned}
$$

where $X(t, x)$ is the particle trajectory satisfying

$$
\left\{\begin{array}{l}
\frac{d}{d t} X(t, x)=u(X(t, x), t), \quad t>0 \\
X(0, x)=x
\end{array}\right.
$$

Integration of (2.6) in time gives

$$
\mu \ln \rho(X(t, x), t)+\xi(X(t, x), t) \leq \mu \ln \rho(x, 0)+\xi(x, 0) .
$$

Making use of (1.5), (2.2), (2.4), and (2.5), we find

$$
\begin{aligned}
\mu \ln \rho(X(t, x), t) & \leq \mu \ln \rho(x, 0)+\xi(x, 0)+\xi(X(t, x), t) \\
& \leq \mu \ln \rho_{0}(x)+\int_{\mathbb{R}} \rho_{0}\left|u_{0}\right| d x+\int_{\mathbb{R}} \rho|u| d x
\end{aligned}
$$




$$
\begin{aligned}
& \leq \mu \rho_{0}+\left\|\rho_{0}^{1 / 2} u_{0}\right\|_{L^{2}}\left\|\rho_{0}\right\|_{L^{1}}^{1 / 2}+\left\|\rho^{1 / 2} u\right\|_{L^{2}}\|\rho\|_{L^{1}}^{1 / 2} \\
& \leq C .
\end{aligned}
$$

This finishes the proof.

Lemma 2.3 We have

$$
\left\|u_{x}\right\|_{L^{2}}+\int_{0}^{T}\left\|\rho^{1 / 2} \dot{u}\right\|_{L^{2}}^{2} d t \leq C(T) .
$$

Proof Multiplying $(1.1)_{2}$ by $\dot{u}$ and integrating by parts give rise to

$$
\begin{aligned}
\frac{d}{d t} & \int_{\mathbb{R}} \frac{\mu}{2}\left|u_{x}\right|^{2} d x+\int_{\mathbb{R}} \rho|\dot{u}|^{2} d x \\
& =\int_{\mathbb{R}} P \dot{u}_{x} d x-\mu \int_{\mathbb{R}} u_{x}\left(u u_{x}\right)_{x} d x \\
& =\int_{\mathbb{R}} P \dot{u}_{x} d x-\frac{\mu}{2} \int_{\mathbb{R}} u_{x}^{3} d x \\
& =\frac{d}{d t} \int_{\mathbb{R}} P u_{x} d x-\int_{\mathbb{R}}\left(P_{t}+(P u)_{x}\right) u_{x} d x+\int_{\mathbb{R}} P u_{x}^{2} d x-\frac{\mu}{2} \int_{\mathbb{R}} u_{x}^{3} d x \\
& =\frac{d}{d t} \int_{\mathbb{R}} P u_{x} d x+\gamma \int_{\mathbb{R}} P u_{x}^{2} d x-\frac{\mu}{2} \int_{\mathbb{R}} u_{x}^{3} d x,
\end{aligned}
$$

where the last equality comes from

$$
P_{t}+P_{x} u+\gamma P u_{x}=0
$$

owing to (1.1) $)_{1}$. By virtue of (2.2), (2.3), and the Sobolev inequality, it satisfies

$$
\begin{aligned}
& \left\|u_{x}\right\|_{L^{\infty}}+\|P\|_{L^{\infty}} \\
& \quad \leq C\left\|\mu u_{x}-P\right\|_{L^{\infty}}+C\|P\|_{L^{\infty}} \\
& \quad \leq C\left\|u_{x}\right\|_{L^{2}}+C\left\|\left(\mu u_{x}-P\right)_{x}\right\|_{L^{2}}+C\|P\|_{L^{2}}+C\|P\|_{L^{\infty}} \\
& \quad \leq C\left(\left\|u_{x}\right\|_{L^{2}}+\left\|\rho^{1 / 2} \dot{u}\right\|_{L^{2}}\right)+C .
\end{aligned}
$$

So, it follows from (2.8) that

$$
\begin{aligned}
\frac{d}{d t} B(t)+\left\|\rho^{1 / 2} \dot{u}\right\|_{L^{2}}^{2} & \leq C\left(1+\left\|u_{x}\right\|_{L^{\infty}}+\|P\|_{L^{\infty}}\right)\left\|u_{x}\right\|_{L^{2}}^{2} \\
& \leq C\left\|u_{x}\right\|_{L^{2}}^{2}+C\left\|u_{x}\right\|_{L^{2}}^{4}+\frac{1}{2}\left\|\rho^{1 / 2} \dot{u}\right\|_{L^{2}}^{2},
\end{aligned}
$$

where

$$
B(t)=\int_{\mathbb{R}}\left(\frac{\mu}{2}\left|u_{x}\right|^{2}-P u_{x}\right) d x
$$

satisfies

$$
\frac{\mu}{4}\left\|u_{x}\right\|_{L^{2}}^{2}-C\|P\|_{L^{2}}^{2} \leq B(t) \leq \mu\left\|u_{x}\right\|_{L^{2}}^{2}+C\|P\|_{L^{2}}^{2} .
$$


Noting (2.2), (2.3), and (2.13), we integrate (2.11) to get

$$
\left\|u_{x}\right\|_{L^{2}(\mathbb{R})}^{2}+\int_{0}^{T}\left\|\rho^{1 / 2} \dot{u}\right\|_{L^{2}(\mathbb{R})}^{2} d t \leq C(T)+C \int_{0}^{T}\left\|u_{x}\right\|_{L^{2}(\mathbb{R})}^{4} d t
$$

which completes the proof in view of the Gronwall inequality and (2.2).

Lemma 2.4 For all $t \in[0,+\infty)$,

$$
\int_{-A}^{A} \rho(x, t) d x \geq \frac{1}{4}
$$

where $A=N_{0}(1+t) \ln (e+t)$, and $N_{0}$ is taken from (2.1).

Proof The idea is borrowed from [17]. Using (2.2) and (2.4), we multiply $(1.1)_{1}$ by $|x|$ to obtain

$$
\frac{d}{d t} \int_{\mathbb{R}} \rho|x| d x \leq C \int_{\mathbb{R}} \rho|u| d x \leq C\|\rho\|_{L^{1}}^{1 / 2}\left\|\rho^{1 / 2} u\right\|_{L^{2}} \leq C .
$$

This together with (1.5) brings about

$$
\int_{\mathbb{R}} \rho(x, t)|x| d x \leq C(1+t)
$$

Define a cut-off function $\varphi_{1}(x) \in C_{0}^{1}(\mathbb{R})$ satisfying

$$
0 \leq \varphi_{1}(x) \leq 1, \quad \varphi_{1}(x)=\left\{\begin{array}{ll}
1, & |x|<1, \\
0, & |x|>2,
\end{array} \quad\left|\varphi_{1}^{\prime}\right| \leq C\right.
$$

Multiplied by $\varphi_{1}(y)$ with $y=\eta x[(1+t) \ln (e+t)]^{-1}$, from $(1.1)_{1}$

$$
\begin{aligned}
\frac{d}{d t} \int_{\mathbb{R}} \rho \varphi_{1}(y) d x & =\int_{\mathbb{R}} \rho \varphi_{1}^{\prime} y_{t} d x+\int_{\mathbb{R}} \rho u \varphi_{1}^{\prime} y_{x} d x \\
& \geq-\frac{2 \eta}{(1+t)^{2} \ln (e+t)} \int_{\mathbb{R}} \rho|x| d x-\frac{\eta}{(1+t) \ln (e+t)} \int_{\mathbb{R}} \rho|u| d x \\
& \geq-\frac{C \eta}{(1+t) \ln (e+t)}
\end{aligned}
$$

where the last inequality is due to (2.15) and (2.2). Integrating the above inequality in time we conclude that

$$
\int_{\mathbb{R}} \rho \varphi_{1}(y) d x \geq \int_{\mathbb{R}} \rho_{0} \varphi_{1}(\eta x) d x-C \eta \geq \frac{1}{4}
$$

This proves (2.14) by choosing $\eta=\left(N_{0}+4 C\right)^{-1}$.

Our next object is to derive some weighted $L^{p}$ estimate on $u$, which plays a key role in our analysis. 
Lemma 2.5 We have for all $p \in[2, \infty)$

$$
\sup _{0 \leq t \leq T}\left\|u \bar{x}^{-2 / p}\right\|_{L^{p}}^{p} \leq C(p, T)\left(\left\|\rho^{1 / p} u\right\|_{L^{p}}^{p}+\left\|\left(u^{p / 2}\right)_{x}\right\|_{L^{2}}^{2}\right) .
$$

Proof Multiplying $(1.1)_{2}$ by $p|u|^{p-2} u$ with $p \geq 2$, we obtain

$$
\begin{aligned}
& \frac{d}{d t} \int_{\mathbb{R}} \rho|u|^{p} d x+\frac{4 \mu(p-1)}{p} \int_{\mathbb{R}}\left|\left(u^{p / 2}\right)_{x}\right|^{2} d x \\
& \quad=2(p-1) \int_{\mathbb{R}} P u^{(p-2) / 2}\left(u^{p / 2}\right)_{x} d x \\
& \quad \leq \frac{2 \mu(p-1)}{p} \int_{\mathbb{R}}\left|\left(u^{p / 2}\right)_{x}\right|^{2} d x+C(p) \int_{\mathbb{R}} P^{2}|u|^{p-2} d x \\
& \quad \leq \frac{2 \mu(p-1)}{p} \int_{\mathbb{R}}\left|\left(u^{p / 2}\right)_{x}\right|^{2} d x+C(p) \int_{\mathbb{R}} \rho|u|^{p} d x+C,
\end{aligned}
$$

where in the last inequality we have used (2.2) and (2.3). Integrating in time leads to

$$
\left\|\rho^{1 / p} u\right\|_{L^{p}}+\int_{0}^{T}\left\|\left(u^{p / 2}\right)_{x}\right\|_{L^{2}}^{2} d t \leq C(T, p)
$$

Let

$$
\overline{u^{p}}=\frac{1}{2 N_{0}} \int_{-N_{0}}^{N_{0}} u^{p} d x
$$

On account of the Poincaré inequality and (2.14), we infer

$$
\begin{aligned}
\overline{u^{p}} & \leq 4 \int_{-N_{0}}^{N_{0}} \rho \overline{u^{p}} d x \leq 4 \int_{-N_{0}}^{N_{0}} \rho\left|u^{p}-\overline{u^{p}}\right| d x+4 \int_{-N_{0}}^{N_{0}} \rho|u|^{p} d x \\
& \leq C \int_{-N_{0}}^{N_{0}}\left|u^{p}-\overline{u^{p}}\right| d x+C \int_{\mathbb{R}} \rho|u|^{p} d x \\
& \leq \frac{1}{2} \overline{u^{p}}+C\left(N_{0}\right) \int_{\mathbb{R}}\left|\left(u^{p / 2}\right)_{x}\right|^{2} d x+C \int_{\mathbb{R}} \rho|u|^{p} d x,
\end{aligned}
$$

that is,

$$
\int_{-N_{0}}^{N_{0}} u^{p} d x \leq C\left(N_{0}\right) \int_{\mathbb{R}}\left|\left(u^{p / 2}\right)_{x}\right|^{2} d x+C\left(N_{0}\right) \int_{\mathbb{R}} \rho|u|^{p} d x .
$$

Next, a straight computation shows for an even number $p=2,4, \ldots$

$$
\begin{aligned}
2 \int_{\mathbb{R}} \frac{\left(1-\varphi_{1}\right) u^{p}}{x^{2} \ln ^{2}|x|} d x & \leq \int_{\mathbb{R}}\left(\frac{-1}{x \ln |x|}\right)^{\prime}\left(1-\varphi_{1}\right) u^{p} d x \\
& =2 \int_{\mathbb{R}} \frac{\left(1-\varphi_{1}\right)}{x \ln |x|} u^{p / 2}\left(u^{p / 2}\right)_{x} d x-\int_{\mathbb{R}} \frac{\varphi_{1}^{\prime}}{x \ln |x|} u^{p} d x \\
& \leq \int_{\mathbb{R}} \frac{\left(1-\varphi_{1}\right) u^{p}}{x^{2} \ln ^{2}|x|} d x+C \int_{\mathbb{R}}\left|\left(u^{p / 2}\right)_{x}\right|^{2} d x+C \int_{\{1 \leq|x| \leq 2\}}|u|^{p} d x,
\end{aligned}
$$


where $\varphi_{1}$ is defined in (2.16). This together with (2.19) gives rise to

$$
\int_{\mathbb{R}} \frac{\left(1-\varphi_{1}\right) u^{p}}{x^{2} \ln ^{2}|x|} d x \leq C \int_{\mathbb{R}}\left|\left(u^{p / 2}\right)_{x}\right|^{2} d x+C \int_{\mathbb{R}} \rho|u|^{p} d x, \quad p=2,4, \ldots
$$

In terms of the Cauchy inequality, (2.20) holds true for all $p \geq 2$.

Lemma 2.6 We have

$$
\sup _{0 \leq t \leq T}\left\|\bar{x}^{a} \rho\right\|_{L^{1} \cap H^{1}} \leq C(T)
$$

Proof It follows from $(1.1)_{1}$ that

$$
\left(\rho \bar{x}^{a}\right)_{t}+\left(u \rho \bar{x}^{a}\right)_{x}=a u \rho \bar{x}^{a}(\ln \bar{x})_{x} .
$$

Observe (2.18) and the following simple facts:

$$
\begin{aligned}
& \left|\partial_{x} \bar{x}\right|+\left|\partial_{x x} \bar{x}\right| \leq C \ln \left(e+x^{2}\right), \\
& \left\|\bar{x}^{(a-p) / p} \ln \left(e+x^{2}\right)\right\|_{L^{\infty}} \leq C, \quad \text { for } a<p,
\end{aligned}
$$

and integrate (2.22) to obtain for $p \geq 2$

$$
\begin{aligned}
\frac{d}{d t} \int_{\mathbb{R}} \rho \bar{x}^{a} d x & \leq C \int_{\mathbb{R}} \rho|u| \bar{x}^{(a-1)} \ln \left(e+x^{2}\right) d x \\
& \leq C\left\|\rho^{1 / p} u\right\|_{L^{p}}\left\|\rho^{(p-1) / p} \bar{x}^{(a-1)} \ln \left(e+x^{2}\right)\right\|_{L^{p /(p-1)}} \\
& \leq C\left\|\rho^{1 / p} u\right\|_{L^{p}}\left\|\bar{x}^{(a-p) / p} \ln \left(e+x^{2}\right)\right\|_{L^{\infty}}\left(\int_{\mathbb{R}} \rho \bar{x}^{a} d x\right)^{(p-1) / p} \\
& \leq C\left(\int_{\mathbb{R}} \rho \bar{x}^{a} d x\right)^{(p-1) / p}
\end{aligned}
$$

and hence

$$
\sup _{0 \leq t \leq T}\left\|\rho \bar{x}^{a}\right\|_{L^{1}} \leq C(T, p) .
$$

Differentiating (2.22) in variable $x$ once more we get

$$
\begin{aligned}
& \left(\rho \bar{x}^{a}\right)_{x t}+u\left(\rho \bar{x}^{a}\right)_{x x}+2 u_{x}\left(\rho \bar{x}^{a}\right)_{x}+\rho \bar{x}^{a}\left(u_{x x}-\mu^{-1} P_{x}\right) \\
& \quad=-\mu^{-1} P_{x} \rho \bar{x}^{a}+a\left[u_{x} \rho \bar{x}^{a}(\ln \bar{x})_{x}+u\left(\rho \bar{x}^{a}\right)_{x}(\ln \bar{x})_{x}+u \rho \bar{x}^{a}(\ln \bar{x})_{x x}\right] .
\end{aligned}
$$

Notice that

$$
P_{x} \rho \bar{x}^{a}=K \gamma \rho^{\gamma}\left[\left(\rho \bar{x}^{a}\right)_{x}-a \rho \bar{x}^{a}(\ln \bar{x})_{x}\right]
$$

and

$$
\left\|u_{x x}-\mu^{-1} P_{x}\right\|_{L^{2}} \leq C\|\rho \dot{u}\|_{L^{2}} \leq C\left\|\rho^{1 / 2} \dot{u}\right\|_{L^{2}},
$$


it satisfies from (2.25) after multiplication by $2\left(\rho \bar{x}^{a}\right)_{x}$

$$
\begin{aligned}
\frac{d}{d t}\left\|\left(\rho \bar{x}^{a}\right)_{x}\right\|_{L^{2}} \leq & C\left(1+\left\|u_{x}\right\|_{L^{\infty}}+\left\|u(\ln \bar{x})_{x}\right\|_{L^{\infty}}\right)\left\|\left(\rho \bar{x}^{a}\right)_{x}\right\|_{L^{2}} \\
& +C\left\|\rho \bar{x}^{a}\right\|_{L^{\infty}}\left\|\rho^{1 / 2} \dot{u}\right\|_{L^{2}} \\
& +C\left\|\rho \bar{x}^{a}\right\|_{L^{2}}\left(1+\left\|u_{x}(\ln \bar{x})_{x}\right\|_{L^{\infty}}+\left\|u(\ln \bar{x})_{x x}\right\|_{L^{\infty}}\right) .
\end{aligned}
$$

Inequalities (2.7), (2.17)-(2.18), and (2.23) ensure that for $p \in(2, \infty)$

$$
\begin{aligned}
& \left\|u(\ln \bar{x})_{x}+u(\ln \bar{x})_{x x}\right\|_{L^{\infty}} \\
& \quad \leq C(p)\left\|u \bar{x}^{-2 / p}\right\|_{L^{\infty}} \\
& \quad \leq C(p)\left(\left\|u \bar{x}^{-2 / p}\right\|_{L^{p}}+\left\|\left(u \bar{x}^{-2 / p}\right)_{x}\right\|_{L^{2}}\right) \\
& \quad \leq C(p, T)\left(\left\|\rho^{1 / p} u\right\|_{L^{p}}+\left\|\left(u^{p / 2}\right)_{x}\right\|_{L^{2}}^{2 / p}+\left\|u_{x}\right\|_{L^{2}}+\left\|u \bar{x}^{-1}\right\|_{L^{2}}\right) \\
& \quad \leq C(p, T)\left(1+\left\|\left(u^{p / 2}\right)_{x}\right\|_{L^{2}}^{2 / p}\right)
\end{aligned}
$$

and that for $p=2$

$$
\left\|u \bar{x}^{-1}\right\|_{L^{\infty}} \leq C(T)\left(\left\|\rho^{1 / 2} u\right\|_{L^{2}}+\left\|u_{x}\right\|_{L^{2}}\right) \leq C(T) .
$$

Thanks to (2.27), (2.7), (2.10), and (2.24), we conclude from (2.26) that

$$
\frac{d}{d t}\left\|\left(\rho \bar{x}^{a}\right)_{x}\right\|_{L^{2}} \leq C\left(1+\left\|\rho^{1 / 2} \dot{u}\right\|_{L^{2}}+\left\|\left(u^{p / 2}\right)_{x}\right\|_{L^{2}}\right)\left(1+\left\|\left(\rho \bar{x}^{a}\right)_{x}\right\|_{L^{2}}\right),
$$

which implies by using (2.7), (2.18), and the Gronwall inequality

$$
\left\|\left(\rho \bar{x}^{a}\right)_{x}\right\|_{L^{2}} \leq C(T)
$$

This together with (2.24) finishes the proof.

\section{Lemma 2.7 We have}

$$
\left\|\rho^{1 / 2} \dot{u}\right\|_{L^{2}}+\int_{0}^{T}\left\|\dot{u}_{x}\right\|_{L^{2}}^{2} d t \leq C(T)
$$

and

$$
\left\|u_{x}\right\|_{L^{\infty}}+\left\|u_{x x}\right\|_{L^{2}} \leq C(T)
$$

Proof Operating $\partial_{t}+\partial_{x}(u \cdot)$ to $(1.1)_{2}$, using (2.9) and (1.1) $)_{1}$, we obtain

$$
\begin{aligned}
\rho \dot{u}_{t}+\rho u \dot{u}_{x}-\mu \dot{u}_{x x} & =-\mu\left(\left|u_{x}\right|^{2}\right)_{x}-\left(P_{t}+(P u)_{x}\right)_{x}+\left(u_{x} P\right)_{x} \\
& =-\mu\left(\left|u_{x}\right|^{2}\right)_{x}+\gamma\left(u_{x} P\right)_{x} .
\end{aligned}
$$


Integration of (2.31) after multiplication by $\dot{u}$ leads to

$$
\frac{1}{2} \frac{d}{d t}\left\|\rho^{1 / 2} \dot{u}\right\|_{L^{2}}^{2}+\mu\left\|\dot{u}_{x}\right\|_{L^{2}}^{2}=\mu \int_{\mathbb{R}} \dot{u}_{x}\left|u_{x}\right|^{2} d x-\gamma \int_{\mathbb{R}} \dot{u}_{x} u_{x} P d x
$$

which combining with (2.2), (2.3), (2.7), and (2.10) lets us conclude that

$$
\begin{aligned}
\frac{d}{d t}\left\|\rho^{1 / 2} \dot{u}\right\|_{L^{2}}^{2}+\left\|\dot{u}_{x}\right\|_{L^{2}}^{2} & \leq C\left\|u_{x}\right\|_{L^{4}}^{4}+C\|P\|_{L^{4}}^{4} \\
& \leq C\left\|u_{x}\right\|_{L^{\infty}}^{2}\left\|u_{x}\right\|_{L^{2}}^{2}+C\|P\|_{L^{1}} \\
& \leq C\left\|\rho^{1 / 2} \dot{u}\right\|_{L^{2}}^{2}+C .
\end{aligned}
$$

Remembering (1.6), we integrate (2.32) to obtain (2.29).

Next, by virtue of (2.21) and (2.29), it follows from $(1.1)_{2}$ that

$$
\begin{aligned}
\left\|u_{x x}\right\|_{L^{2}} & \leq C(\mu)\left\|u_{x x}-\mu^{-1} P_{x}\right\|_{L^{2}}+\left\|P_{x}\right\|_{L^{2}} \\
& \leq C\left\|\rho^{1 / 2} \dot{u}\right\|_{L^{2}}+C\left\|\rho \bar{x}^{a}\right\|_{H^{1}} \leq C,
\end{aligned}
$$

which together with (2.10) yields (2.30).

\section{A priori estimates (II)}

In this section, we derive the higher-order regularity estimates of the solutions.

\section{Lemma 3.1 We have}

$$
\sup _{0 \leq t \leq T} t\left\|\dot{u}_{x}\right\|_{L^{2}}^{2}+\int_{0}^{T} t\left(\left\|\rho^{1 / 2} \ddot{u}\right\|_{L^{2}}^{2}+\left\|\dot{u}_{x x}\right\|_{L^{2}}^{2}\right) d t \leq C(T) .
$$

Proof Multiplying (2.31) by $\ddot{u}$ leads to

$$
\frac{1}{2} \int_{\mathbb{R}} \rho|\ddot{u}|^{2} d x=\mu \int_{\mathbb{R}} \dot{u}_{x x} \ddot{u} d x-\mu \int_{\mathbb{R}}\left(\left|u_{x}\right|^{2}\right)_{x} \ddot{u} d x+\gamma \int_{\mathbb{R}}\left(u_{x} P\right)_{x} \ddot{u} d x .
$$

We estimate the terms on the right-hand side of (3.2) as follows. First,

$$
\mu \int_{\mathbb{R}} \dot{u}_{x x} \ddot{u} d x=\mu \int_{\mathbb{R}} \dot{u}_{x x}\left(\partial_{t} \dot{u}+u \dot{u}_{x}\right) d x=-\frac{\mu}{2} \frac{d}{d t} \int_{\mathbb{R}} \dot{u}_{x}^{2}-\frac{\mu}{2} \int_{\mathbb{R}} \dot{u}_{x}^{2} u_{x} d x .
$$

Second,

$$
\begin{aligned}
& -\mu \int_{\mathbb{R}}\left(\left|u_{x}\right|^{2}\right)_{x} \ddot{u} d x \\
& \quad=\mu \int_{\mathbb{R}}\left|u_{x}\right|^{2}\left(\dot{u}_{t}+u \dot{u}_{x}\right)_{x} d x \\
& \quad=\mu \frac{d}{d t} \int_{\mathbb{R}}\left|u_{x}\right|^{2} \dot{u}_{x} d x-2 \mu \int_{\mathbb{R}} u_{x}\left(\dot{u}-u u_{x}\right)_{x} \dot{u}_{x} d x+\mu \int_{\mathbb{R}}\left|u_{x}\right|^{2}\left(u \dot{u}_{x}\right)_{x} d x \\
& =\mu \frac{d}{d t} \int_{\mathbb{R}}\left|u_{x}\right|^{2} \dot{u}_{x} d x-2 \mu \int_{\mathbb{R}} u_{x} \dot{u}_{x}^{2} d x
\end{aligned}
$$




$$
\begin{aligned}
& +2 \mu \int_{\mathbb{R}} u_{x}\left(u u_{x}\right)_{x} \dot{u}_{x} d x+\mu \int_{\mathbb{R}}\left|u_{x}\right|^{2}\left(u \dot{u}_{x}\right)_{x} d x \\
= & \mu \frac{d}{d t} \int_{\mathbb{R}}\left|u_{x}\right|^{2} \dot{u}_{x} d x-2 \mu \int_{\mathbb{R}} u_{x} \dot{u}_{x}^{2} d x+2 \mu \int_{\mathbb{R}}\left(u_{x}\right)^{3} \dot{u}_{x} d x .
\end{aligned}
$$

By (2.9), the final term becomes

$$
\begin{aligned}
\int_{\mathbb{R}} & \left(u_{x} P\right)_{x} \ddot{u} d x \\
= & -\int_{\mathbb{R}} u_{x} P\left(\dot{u}_{t}+u \dot{u}_{x}\right)_{x} d x \\
= & -\frac{d}{d t} \int_{\mathbb{R}} u_{x} P \dot{u}_{x} d x+\int_{\mathbb{R}}\left(\dot{u}_{x}^{2} P+\dot{u}_{x} u_{x}\left[P_{t}+(u P)_{x}-P u_{x}\right]\right) d x \\
& +\int_{\mathbb{R}} \dot{u}_{x x} P u u_{x} d x-\int_{\mathbb{R}} P u_{x}\left(u \dot{u}_{x}\right)_{x} d x \\
= & -\frac{d}{d t} \int_{\mathbb{R}} u_{x} P \dot{u}_{x} d x+\int_{\mathbb{R}}\left(\dot{u}_{x}^{2} P-\gamma \dot{u}_{x} u_{x} P u_{x}\right) d x-\int_{\mathbb{R}} \dot{u}_{x} P u_{x}^{2} d x .
\end{aligned}
$$

Substitute the last three inequalities into (3.2), take (2.2), (2.3), and (2.30) into account, and we arrive at

$$
\begin{aligned}
& \frac{d}{d t} I(t)+\frac{1}{2} \int_{\mathbb{R}} \rho|\ddot{u}|^{2} d x \\
& \quad \leq C\left(1+\left\|u_{x}\right\|_{L^{\infty}}+\|P\|_{L^{\infty}}\right)\left\|\dot{u}_{x}\right\|_{L^{2}}^{2}+C\left(\left\|u_{x}\right\|_{L^{6}}^{6}+\|P\|_{L^{6}}^{6}\right) \\
& \quad \leq C\left\|\dot{u}_{x}\right\|_{L^{2}}^{2}+C,
\end{aligned}
$$

where

$$
I(t)=\int_{\mathbb{R}}\left(\frac{\mu}{2}\left|\dot{u}_{x}\right|^{2}-\mu\left|u_{x}\right|^{2} \dot{u}_{x}+\gamma P u_{x} \dot{u}_{x}\right) d x
$$

satisfies

$$
\frac{\mu}{4}\left\|\dot{u}_{x}\right\|_{L^{2}}^{2}-C \leq I(t) \leq C\left\|\dot{u}_{x}\right\|_{L^{2}}^{2}+C .
$$

Thanks to (2.29), we multiply (3.3) by $t$ and integrate the resulting expression to deduce

$$
\sup _{0 \leq t \leq T} t\left\|\dot{u}_{x}\right\|_{L^{2}}^{2}+\int_{0}^{T} t\left\|\rho^{1 / 2} \ddot{u}\right\|_{L^{2}}^{2} d t \leq C(T) .
$$

It follows from (2.31) that

$$
\begin{aligned}
\left\|\dot{u}_{x x}\right\|_{L^{2}} & \leq C\|\rho \ddot{u}\|_{L^{2}}+C\left(\left\|u_{x}\right\|_{L^{\infty}}+\|P\|_{L^{\infty}}\right)\left(\left\|u_{x x}\right\|_{L^{2}}+\left\|P_{x}\right\|_{L^{2}}\right) \\
& \leq C\left\|\rho^{1 / 2} \ddot{u}\right\|_{L^{2}}+C\left\|u_{x x}\right\|_{L^{2}}+C\left\|\rho \bar{x}^{a}\right\|_{H^{1}} \\
& \leq C\left(\left\|\rho^{1 / 2} \ddot{u}\right\|_{L^{2}}+1\right),
\end{aligned}
$$


in which (2.2), (2.3), (2.21), and (2.30) have been used. Integrating (3.5) gives

$$
\int_{0}^{T} t\left\|\dot{u}_{x x}\right\|_{L^{2}}^{2} d t \leq C(T)
$$

owing to (3.4).

Lemma 3.2 We have

$$
\sup _{0 \leq t \leq T}\left(\left\|\rho_{x x}\right\|_{L^{2}}+\left\|P_{x x}\right\|_{L^{2}}+t\left\|u_{x x x}\right\|_{L^{2}}^{2}\right) \leq C(T) .
$$

Proof Differentiating $(1.1)_{1}$ with respect to $x$ twice we get

$$
\rho_{x x t}=-u \rho_{x x x}-3 u_{x} \rho_{x x}-3 u_{x x} \rho_{x}-u_{x x x} \rho,
$$

which satisfies after multiplication by $2 \rho_{x x}$,

$$
\begin{aligned}
\frac{d}{d t}\left\|\rho_{x x}\right\|_{L^{2}} & \leq C\left\|u_{x}\right\|_{L^{\infty}}\left\|\rho_{x x}\right\|_{L^{2}}+C\left\|\rho_{x}\right\|_{L^{\infty}}\left\|u_{x x}\right\|_{L^{2}}+C\|\rho\|_{L^{\infty}}\left\|u_{x x x}\right\|_{L^{2}} \\
& \leq C\left\|\rho_{x x}\right\|_{L^{2}}+C\left\|u_{x x x}\right\|_{L^{2}}+C
\end{aligned}
$$

where the last inequality follows from (2.3), (2.21), and (2.30).

A similar argument to (3.7) lets us deduce from (2.9) that

$$
\frac{d}{d t}\left\|P_{x x}\right\|_{L^{2}} \leq C\left\|P_{x x}\right\|_{L^{2}}+C\left\|u_{x x x}\right\|_{L^{2}}+C .
$$

In order to examine $\left\|u_{x x x}\right\|_{L^{2}}$, we differentiate $(1.1)_{2}$ and obtain

$$
\mu u_{x x x}=\rho_{x} \dot{u}+\rho \dot{u}_{x}+P_{x x}
$$

which, along with (2.3), (2.21), (2.28), and (2.29), leads us to conclude

$$
\begin{aligned}
\left\|u_{x x x}\right\|_{L^{2}} & \leq C\left(\left\|\rho_{x} \dot{u}\right\|_{L^{2}}+\left\|\rho \dot{u}_{x}\right\|_{L^{2}}+\left\|P_{x x}\right\|_{L^{2}}\right) \\
& \leq C\left(\left\|\rho \bar{x}^{a}\right\|_{H^{1}}\left\|\bar{x}^{-a} \dot{u}\right\|_{L^{\infty}}+\left\|\dot{u}_{x}\right\|_{L^{2}}+\left\|P_{x x}\right\|_{L^{2}}\right) \\
& \leq C\left(1+\left\|\dot{u}_{x}\right\|_{L^{2}}+\left\|P_{x x}\right\|_{L^{2}}\right) .
\end{aligned}
$$

Once (3.9) is obtained, inequalities (3.7) and (3.8) imply that

$$
\frac{d}{d t}\left(\left\|\rho_{x x}\right\|_{L^{2}}+\left\|P_{x x}\right\|_{L^{2}}\right) \leq C\left(\left\|\rho_{x x}\right\|_{L^{2}}+\left\|P_{x x}\right\|_{L^{2}}\right)+C\left\|\dot{u}_{x}\right\|_{L^{2}}+C,
$$

which generates by using (2.29) and the Gronwall inequality

$$
\left\|\rho_{x x}\right\|_{L^{2}}+\left\|P_{x x}\right\|_{L^{2}} \leq C(T) \text {. }
$$

As a result of (3.10) and (3.1), we have from (3.9)

$$
t\left\|u_{x x x}\right\|_{L^{2}}^{2} \leq C(T) .
$$

This inequality as well as (3.10) completes the proof. 
Lemma 3.3 We have

$$
\sup _{0 \leq t \leq T} t\left(\left\|\rho^{1 / 2} \ddot{u}\right\|_{L^{2}}+\left\|\dot{u}_{x x}\right\|_{L^{2}}\right)+\int_{0}^{T} t^{2}\left\|\ddot{u}_{x}\right\|_{L^{2}}^{2} d t \leq C .
$$

Proof Utilizing $(1.1)_{1}$ and (2.9), operating by $\partial_{t}+\partial_{x}(u \cdot)$ to (2.31), it yields after a straightforward but tedious calculation

$$
\rho \ddot{u}_{t}+\rho u \ddot{u}_{x}-\mu \ddot{u}_{x x}=-3 \mu\left(u_{x} \dot{u}_{x}\right)_{x}+2 \mu\left(u_{x}^{3}\right)_{x}+\gamma\left(P \dot{u}_{x}\right)_{x}-\gamma(\gamma+1)\left(u_{x}^{2} P\right)_{x}
$$

which gives after multiplication by $\ddot{u}$

$$
\begin{aligned}
& \frac{1}{2} \frac{d}{d t} \int_{\mathbb{R}} \rho|\ddot{u}|^{2} d x+\mu \int_{\mathbb{R}}\left|\ddot{u}_{x}\right|^{2} d x \\
& \quad=3 \mu \int_{\mathbb{R}} \ddot{u}_{x} u_{x} \dot{u}_{x} d x-2 \mu \int_{\mathbb{R}} \ddot{u}_{x} u_{x}^{3} d x-\gamma \int_{\mathbb{R}} \ddot{u}_{x} \dot{u}_{x} P d x+\gamma(\gamma+1) \int_{\mathbb{R}} \ddot{u}_{x} u_{x}^{2} P d x,
\end{aligned}
$$

from which, together with (2.3), (2.7), (2.30), and the Cauchy inequality, one deduces

$$
\frac{d}{d t}\left\|\rho^{1 / 2} \ddot{u}\right\|_{L^{2}}^{2}+\left\|\ddot{u}_{x}\right\|_{L^{2}}^{2} \leq C+C\left\|\dot{u}_{x}\right\|_{L^{2}}^{2}
$$

Recalling (3.1), integration of (3.12) and multiplication by $t^{2}$ yields

$$
t^{2}\left\|\rho^{1 / 2} \ddot{u}\right\|_{L^{2}}^{2}+\int_{0}^{T} t^{2}\left\|\ddot{u}_{x}\right\|_{L^{2}}^{2} d t \leq C(T) .
$$

Consequently, it follows from (3.5) that

$$
\sup _{0 \leq t \leq T} t\left\|\dot{u}_{x x}\right\|_{L^{2}} \leq C(T)
$$

The proof is finished.

\section{Proof of Theorem 1.1}

Now we are ready to prove Theorem 1.1. For this we first state the local-in-time existence and uniqueness of the classical solution to (1.1)-(1.3).

Lemma 4.1 Under the hypotheses in Theorem 1.1, the Cauchy problem (1.1)-(1.3) admits a unique classical solution $(\rho, u)$ over $\mathbb{R} \times\left(0, T_{*}\right]$ for some small $T_{*}>0$, which satisfies the properties (1.7).

Proof The existence part is exactly the same procedure as in [16]. For the sake of brevity, we only prove the uniqueness. Assume that $(\rho, u)$ and $(\bar{\rho}, \bar{u})$ are two different solutions with the identical initial data. Minus $(1.1)_{2}$ satisfied by $(\rho, u)$ and $(\bar{\rho}, \bar{u})$ yields

$$
\rho U_{t}+\rho u U_{x}-\mu U_{x x}=-\rho U \bar{u}_{x}-H\left(\bar{u}_{t}+\bar{u} \bar{u}_{x}\right)-(P(\rho)-P(\bar{\rho}))_{x},
$$

with

$$
H=\rho-\bar{\rho}, \quad U=u-\bar{u} .
$$


By virtue of (1.7), we multiply (4.1) by $U$ and conclude

$$
\begin{aligned}
& \frac{d}{d t} \int_{\mathbb{R}} \rho U^{2} d x+\mu \int_{\mathbb{R}} U_{x}^{2} d x \\
& \quad \leq C \int_{\mathbb{R}} \rho U^{2} d x+C \int_{\mathbb{R}}|H||U||\dot{\bar{u}}| d x+C\|P(\rho)-P(\bar{\rho})\|_{L^{2}}^{2},
\end{aligned}
$$

where $\dot{\bar{u}}=\bar{u}_{t}+\bar{u} \bar{u}_{x}$. Hölder's inequality together with (2.28) and (2.29) shows

$$
\begin{aligned}
\int_{\mathbb{R}}|H\|U\| \dot{\bar{u}}| d x & \leq C\left\|\dot{\bar{u}} \bar{x}^{-1}\right\|_{L^{\infty}}\left\|H \bar{x}^{2}\right\|_{L^{2}}\left\|U \bar{x}^{-1}\right\|_{L^{2}} \\
& \leq C(\varepsilon)\left(1+\left\|\dot{\bar{u}}_{x}\right\|_{L^{2}}^{2}\right)\left\|H \bar{x}^{2}\right\|_{L^{2}}^{2}+\varepsilon\left(\left\|\rho^{1 / 2} U\right\|_{L^{2}}^{2}+\left\|U_{x}\right\|_{L^{2}}^{2}\right) .
\end{aligned}
$$

Next, from the mass equation we have

$$
H_{t}+\bar{u} H_{x}+H \bar{u}_{x}+\rho U_{x}+U \rho_{x}=0
$$

Multiplied by $H \bar{x}^{4}$, it satisfies

$$
\begin{aligned}
\frac{d}{d t}\left\|H \bar{x}^{2}\right\|_{L^{2}}^{2} \leq & C\left(\left\|\bar{u}_{x}\right\|_{L^{\infty}}+\left\|\bar{u} \bar{x}^{-1 / 2}\right\|_{L^{\infty}}\right)\left\|H \bar{x}^{2}\right\|_{L^{2}}^{2} \\
& +C\left\|H \bar{x}^{2}\right\|_{L^{2}}\left(\left\|\rho \bar{x}^{2}\right\|_{L^{\infty}}\left\|U_{x}\right\|_{L^{2}}+\left\|U \bar{x}^{-1}\right\|_{L^{\infty}}\left\|\bar{x}^{3} \rho_{x}\right\|_{L^{2}}\right) \\
\leq & C(\varepsilon)\left(1+\left\|\left(\bar{u}^{2}\right)_{x}\right\|_{L^{2}}\right)\left\|H \bar{x}^{2}\right\|_{L^{2}}^{2}+\varepsilon\left(\left\|U_{x}\right\|_{L^{2}}^{2}+\left\|\rho^{1 / 2} U\right\|_{L^{2}}^{2}\right)
\end{aligned}
$$

where the second inequality comes from (2.2), (2.21), (2.27), (2.28), and (2.30).

Remark 4.1 The restriction $a \geq 3$ is from (4.4), i.e.,

$$
\left\|\bar{x}^{3} \rho_{x}\right\|_{L^{2}} \leq C\left\|\bar{x}^{3} \rho\right\|_{H^{1}} \leq C\left\|\bar{x}^{a} \rho\right\|_{H^{1}} \leq C
$$

Substituting (4.2) and (4.3) into (4.4), choosing $\varepsilon$ small, we discover

$$
\begin{aligned}
& \frac{d}{d t}\left(\left\|H \bar{x}^{2}\right\|_{L^{2}}^{2}+\left\|\rho^{1 / 2} U\right\|_{L^{2}}^{2}\right)+\left\|U_{x}\right\|_{L^{2}}^{2} \\
& \quad \leq C\left(1+\left\|\dot{\bar{u}}_{x}\right\|_{L^{2}}+\left\|\left(\bar{u}^{2}\right)_{x}\right\|_{L^{2}}\right)\left(\left\|H \bar{x}^{2}\right\|_{L^{2}}^{2}+\left\|\rho^{1 / 2} U\right\|_{L^{2}}^{2}\right)+C\|P(\rho)-P(\bar{\rho})\|_{L^{2}}^{2}
\end{aligned}
$$

One deduces from (2.9) that

$$
(P(\rho)-P(\bar{\rho}))_{t}+\bar{u}(P(\rho)-P(\bar{\rho}))_{x}+U P(\rho)_{x}+\gamma(P(\rho)-P(\bar{\rho})) \bar{u}_{x}+\gamma P(\rho) U_{x}=0,
$$

which gives rise to

$$
\frac{d}{d t}\|P(\rho)-P(\bar{\rho})\|_{L^{2}} \leq C\|P(\rho)-P(\bar{\rho})\|_{L^{2}}+C\left\|U_{x}\right\|_{L^{2}}+C\left\|\rho^{1 / 2} U\right\|_{L^{2}}
$$

Hence,

$$
\|P(\rho)-P(\bar{\rho})\|_{L^{2}} \leq C \int_{0}^{t}\left(\left\|U_{x}\right\|_{L^{2}}+\left\|\rho^{1 / 2} U\right\|_{L^{2}}\right) d s .
$$


Inserting (4.6) back into (4.5) yields

$$
\frac{d}{d t} G \leq C\left(1+\left\|\dot{\bar{u}}_{x}\right\|_{L^{2}}+\left\|\left(\bar{u}^{2}\right)_{x}\right\|_{L^{2}}\right) G
$$

with

$$
G(t)=\left\|H \bar{x}^{2}\right\|_{L^{2}}+\left\|\rho^{1 / 2} U\right\|_{L^{2}}+\int_{0}^{t}\left(\left\|\rho^{1 / 2} U\right\|_{L^{2}}^{2}+\left\|U_{x}\right\|_{L^{2}}^{2}\right) d s .
$$

In view of (2.18) and (2.29), from the Gronwall inequality one concludes $G(t)=0$, which implies that $H(x, t)=U(x, t)=0$ almost everywhere $(x, t) \in \mathbb{R} \times(0, T)$.

Next we show that the solution exists globally in time. Suppose $T^{*} \geq T_{*}$ is the maximal time of existence. We claim that

$$
T^{*}=\infty
$$

If (4.7) is violated, i.e., $T^{*}<\infty$. Then the a priori estimates in Sections 2 and 3 guarantee that the conditions (1.5) make sense at $T=T^{*}$, and, moreover, the compatibility condition (1.6) could be replaced by

$$
-\mu u_{x x}\left(x, T^{*}\right)+P_{x}\left(x, T^{*}\right)=\rho^{1 / 2}\left(x, T^{*}\right) g^{*}
$$

with $g^{*}=-\rho^{1 / 2} \dot{u}\left(x, T^{*}\right) \in L^{2}$. However, the local existence result Lemma 4.1 tells that $(\rho, u)$ could be extended to $\left(0, T^{*}+\tilde{T}_{*}\right]$ with another small $\tilde{T}_{*}>0$. The contradiction implies that (4.7) is true, and thus Theorem 1.1 is completed.

\section{Proof of Corollary 1.1}

In this final section the generic constant $C$ is independent of $T$. Following [15], we define

$$
A_{1}(T)=\sup _{t \in[0, T]} \sigma\left\|u_{x}\right\|_{L^{2}}^{2}+\int_{0}^{T} \sigma\left\|\rho^{1 / 2} \dot{u}\right\|_{L^{2}}^{2} d t
$$

and

$$
A_{2}(T)=\sup _{t \in[0, T]} \sigma^{2}\left\|\rho^{1 / 2} \dot{u}\right\|_{L^{2}}^{2}+\int_{0}^{T} \sigma^{2}\left\|\dot{u}_{x}\right\|_{L^{2}}^{2} d t
$$

where $\sigma \in \min \{t, 1\}$.

Remember (2.8); one has

$$
\frac{d}{d t} B(t)+\int_{\mathbb{R}} \rho|\dot{u}|^{2} d x=\gamma \int_{\mathbb{R}} P\left|u_{x}\right|^{2} d x-\frac{\mu}{2} \int_{\mathbb{R}} u_{x}^{3} d x,
$$

where $B(t)$ is taken from (2.12). Multiplied by $\sigma$, it yields from (5.1) after integration

$$
\begin{aligned}
A_{1}(T) & \leq C \sigma\|P\|_{L^{2}}^{2}+C \int_{0}^{T} \sigma^{\prime} B(t) d t+C \int_{0}^{T} \sigma \int_{\mathbb{R}}\left(P\left|u_{x}\right|^{2}+\left|u_{x}\right|^{3}\right) d x d t \\
& \leq C\|P\|_{L^{2}}^{2}+C \int_{0}^{T}\left\|u_{x}\right\|_{L^{2}}^{2} d t+C \int_{0}^{T} \sigma \int_{\mathbb{R}}\left|u_{x}\right|^{3} d x d t \\
& \leq C+C \int_{0}^{T} \sigma\left\|u_{x}\right\|_{L^{3}}^{3} d t,
\end{aligned}
$$


where we have used (2.2), (2.3), and (2.13). If we multiply (2.32) by $\sigma^{2}$, we obtain

$$
A_{2}(T) \leq C A_{1}(T)+C \int_{0}^{T} \sigma^{2}\left(\left\|u_{x}\right\|_{L^{4}}^{4}+\|P\|_{L^{4}}^{4}\right) d t .
$$

In view of and $(1.1)_{2}$ and (2.3), the Sobolev inequality ensures that

$$
\begin{aligned}
\left\|u_{x}\right\|_{L^{4}}^{4} & \leq C\left\|\mu u_{x}-P\right\|_{L^{4}}^{4}+C\|P\|_{L^{4}}^{4} \\
& \leq C\|\rho \dot{u}\|_{L^{2}}\left(\left\|u_{x}\right\|_{L^{2}}^{3}+\|P\|_{L^{2}}^{3}\right)+C\|P\|_{L^{4}}^{4} \\
& \leq C\left\|\rho^{1 / 2} \dot{u}\right\|_{L^{2}}\left(\left\|u_{x}\right\|_{L^{2}}^{3}+\|P\|_{L^{2}}^{3}\right)+C\|P\|_{L^{4}}^{4} .
\end{aligned}
$$

By this we estimate

$$
\begin{aligned}
C \int_{0}^{T} \sigma^{2}\left\|u_{x}\right\|_{L^{4}}^{4} d t \leq & C \int_{0}^{T} \sigma^{2}\left[\left\|\rho^{1 / 2} \dot{u}\right\|_{L^{2}}\left(\left\|u_{x}\right\|_{L^{2}}^{3}+\|P\|_{L^{2}}^{3}\right)+\|P\|_{L^{4}}^{4}\right] d t \\
\leq & C A_{2}^{1 / 2}(T) A_{1}^{1 / 2}(T) \int_{0}^{T} \sigma^{1 / 2}\left\|u_{x}\right\|_{L^{2}}^{2} d t \\
& +C A_{1}(T)+\int_{0}^{T} \sigma^{2}\left(\|P\|_{L^{2}}^{6}+\|P\|_{L^{4}}^{4}\right) d t \\
\leq & \frac{1}{2} A_{2}(T)+C A_{1}(T)+\int_{0}^{T} \sigma^{2}\left(\|P\|_{L^{2}}^{6}+\|P\|_{L^{4}}^{4}\right) d t
\end{aligned}
$$

which guarantees that (5.3) satisfies

$$
\begin{aligned}
A_{2}(T) & \leq C A_{1}(T)+\int_{0}^{T} \sigma^{2}\left(\|P\|_{L^{2}}^{6}+\|P\|_{L^{4}}^{4}\right) d t \\
& \leq C+C \int_{0}^{T} \sigma\left\|u_{x}\right\|_{L^{3}}^{3} d t+\int_{0}^{T} \sigma^{2}\left(\|P\|_{L^{2}}^{6}+\|P\|_{L^{4}}^{4}\right) d t
\end{aligned}
$$

where in the second inequality we have used (5.2).

In order to examine the last term on the right hand-side of (5.4), we integrate $(1.1)_{2}$ and obtain

$$
P(x, t)=\mu u_{x}-\int_{-\infty}^{x} \rho \dot{u},
$$

which implies

$$
P(x, t) \leq \mu\left|u_{x}\right|+\|\rho \dot{u}\|_{L^{1}} \leq \mu\left|u_{x}\right|+\left\|\rho^{1 / 2} \dot{u}\right\|_{L^{2}} .
$$

By this we compute

$$
\begin{aligned}
\int_{\mathbb{R}} P^{(2 \gamma+1) / \gamma} & =K^{1 / \gamma} \int_{\mathbb{R}} \rho P^{2} \\
& \leq C \int_{\mathbb{R}} \rho\left|u_{x}\right|^{2}+C \int_{\mathbb{R}} \rho\left\|\rho^{1 / 2} \dot{u}\right\|_{L^{2}}^{2} \\
& \leq C\left\|u_{x}\right\|_{L^{2}}^{2}+C\left\|\rho^{1 / 2} \dot{u}\right\|_{L^{2}}^{2} .
\end{aligned}
$$


Because of $\gamma>1$, combination of (2.3) and (5.5) gives

$$
\begin{aligned}
\|P\|_{L^{3}}^{3} & \leq \int_{\mathbb{R}} P^{(2 \gamma+1) / \gamma} P^{(\gamma-1) / \gamma} \\
& \leq C \int_{\mathbb{R}} P^{(2 \gamma+1) / \gamma} \leq C\left\|u_{x}\right\|_{L^{2}}^{2}+C\left\|\rho^{1 / 2} \dot{u}\right\|_{L^{2}}^{2},
\end{aligned}
$$

and thus

$$
\begin{aligned}
\|P\|_{L^{2}}^{6}+\|P\|_{L^{4}}^{4} & \leq C\|P\|_{L^{4}}^{4}\left(\|P\|_{L^{1}}^{2}+1\right) \\
& \leq C\|P\|_{L^{4}}^{4} \leq C\|P\|_{L^{3}}^{3} \leq C\left\|u_{x}\right\|_{L^{2}}^{2}+C\left\|\rho^{1 / 2} \dot{u}\right\|_{L^{2}}^{2} .
\end{aligned}
$$

Inserting (5.6) into (5.4), using (2.2), (5.2), and the definition of $A_{1}(T)$, we obtain

$$
A_{1}(T)+A_{2}(T) \leq C+C \int_{0}^{T} \sigma\left\|u_{x}\right\|_{L^{3}}^{3} d t
$$

It is only left for us to deal with $\int_{0}^{T} \sigma\left\|u_{x}\right\|_{L^{3}}^{3} d t$. Notice that

$$
\begin{aligned}
\left\|u_{x}\right\|_{L^{r}} & \leq C\left\|\mu u_{x}-P\right\|_{L^{r}}+C\|P\|_{L^{r}} \\
& \leq C\|\rho \dot{u}\|_{L^{1}}^{(r-2) / r}\left(\left\|u_{x}\right\|_{L^{2}}^{2 / r}+\|P\|_{L^{2}}^{2 / r}\right)+C\|P\|_{L^{r}} \\
& \leq C\left\|\rho^{1 / 2} \dot{u}\right\|_{L^{2}}^{(r-2) / r}\left(\left\|u_{x}\right\|_{L^{2}}^{2 / r}+\|P\|_{L^{2}}^{2 / r}\right)+C\|P\|_{L^{r}} \quad(r=3,4) .
\end{aligned}
$$

This, along with (2.2) and (5.5), guarantees that

$$
\begin{aligned}
C \int_{0}^{\sigma(T)} \sigma\left\|u_{x}\right\|_{L^{3}}^{3} d t & \leq C \int_{0}^{\sigma(T)} \sigma\left\|\rho^{1 / 2} \dot{u}\right\|_{L^{2}}\left(\left\|u_{x}\right\|_{L^{2}}^{2}+\|P\|_{L^{2}}^{2}\right) d t+C \\
& \leq C A_{2}^{1 / 2}(\sigma(T)) \int_{0}^{\sigma(T)}\left(\left\|u_{x}\right\|_{L^{2}}^{2}+\|P\|_{L^{2}}^{2}\right) d t+C \\
& \leq C A_{2}^{1 / 2}(\sigma(T))+C \leq \varepsilon A_{2}(T)+C
\end{aligned}
$$

and that

$$
\begin{aligned}
& C \int_{\sigma(T)}^{T} \sigma\left\|u_{x}\right\|_{L^{3}}^{3} d t \\
& \quad \leq \varepsilon \int_{\sigma(T)}^{T}\left\|u_{x}\right\|_{L^{4}}^{4} d t+C(\varepsilon) \int_{\sigma(T)}^{T}\left\|u_{x}\right\|_{L^{2}}^{2} d t \\
& \quad \leq \varepsilon \int_{\sigma(T)}^{T}\left(\left\|\rho^{1 / 2} \dot{u}\right\|_{L^{2}}^{2}\left\|u_{x}\right\|_{L^{2}}^{2}+\left\|\rho^{1 / 2} \dot{u}\right\|_{L^{2}}^{2}\|P\|_{L^{2}}^{2}+\|P\|_{L^{4}}^{4}\right)+C(\varepsilon) \\
& \quad \leq \varepsilon\left(A_{2}(T)+A_{1}(T)+\int_{\sigma(T)}^{T}\|P\|_{L^{4}}^{4}\right)+C(\varepsilon) \\
& \quad \leq \varepsilon\left(A_{2}(T)+A_{1}(T)\right)+C(\varepsilon)
\end{aligned}
$$

where in the last inequality we have used (5.6). 
Substituting (5.9) and (5.10) back into (5.7), and selecting $\varepsilon$ so small as to

$$
A_{1}+A_{2} \leq C
$$

Having (5.11) in hand, we use (2.2), (2.3), and (5.5) to get

$$
\int_{0}^{\infty}\|P\|_{L^{(2 \gamma+1) / \gamma}}^{(2 \gamma+1) / \gamma} d t \leq C+\int_{1}^{\infty}\left\|u_{x}\right\|_{L^{2}}^{2} d t+\int_{1}^{\infty}\left\|\rho^{1 / 2} \dot{u}\right\|_{L^{2}}^{2} d t \leq C .
$$

This together with (2.3) yields (1.9) as desired.

\section{Competing interests}

The authors declare that they have no competing interests.

\section{Authors' contributions}

The main idea of this paper was proposed by $Z \mathrm{~L}$, and $\mathrm{YL}$ prepared the manuscript initially and performed all the steps of the proofs in this research. We all have read and approved the final manuscript.

\section{Author details}

${ }^{1}$ School of Economic Mathematics, Southwestern University of Finance and Economics, Chengdu, 611130, P.R. China. ${ }^{2}$ School of International Trade and Economics, University of International Business and Economics, Beijing, 100029,

P.R. China.

\section{Acknowledgements}

The work is partially supported by National Natural Sciences Foundation of China No. 11301422.

Received: 29 December 2014 Accepted: 30 May 2015 Published online: 17 June 2015

\section{References}

1. Hoff, D: Global existence of the Navier-Stokes equations for multidimensional compressible flow with discontinuous initial data. J. Differ. Equ. 120, 215-254 (1995)

2. Hoff, D: Discontinuous solutions of the Navier-Stokes equations for multi-dimensional heat-conducting fluids. Arch. Ration. Mech. Anal. 193, 303-354 (1997)

3. Matsumura, A, Nishida, T: The initial value problem for the equations of motion of viscous and heat-conductive gases. J. Math. Kyoto Univ. 20(1), 67-104 (1980)

4. Kazhikhov, A, Shelukhin, V: Unique global solution with respect to time of initial-boundary value problems for one-dimensional equations of a viscous gas. Prikl. Mat. Meh. 41, 282-291 (1977)

5. Lions, P: Mathematical Topics in Fluid Mechanics: Volume 2: Compressible Models. Oxford Science Publication, Oxford (1998)

6. Feireisl, E: Dynamics of Viscous Compressible Fluids. Oxford University Press, Oxford (2004)

7. Desjardins, B: Regularity of weak solutions of the compressible isentropic Navier-Stokes equations. Commun. Partial Differ. Equ. 22(5-6), 977-1008 (1997)

8. Hoff, D: Compressible flow in a half-space with Navier boundary conditions. J. Math. Fluid Mech. 7, 315-338 (2005)

9. Cho, Y, Choe, H, Kim, H: Unique solvability of the initial boundary value problems for compressible viscous fluids. J. Math. Pures Appl. 83(9), 243-275 (2004)

10. Choe, H, Kim, H: Strong solutions of the Navier-Stokes equations for isentropic compressible fluids. J. Differ. Equ. 190, 504-523 (2003)

11. Cho, Y, Kim, H: On classical solutions of the compressible Navier-Stokes equations with nonnegative initial densities Manuscr. Math. 120, 91-129 (2006)

12. Ding, S, Wen, H, Zhu, C: Global classical large solutions to 1D compressible Navier-Stokes equations with density-dependent viscosity and vacuum. J. Differ. Equ. 251, 1696-1725 (2011)

13. Choe, $\mathrm{H}, \mathrm{Kim}, \mathrm{H}$ : Global existence of the radially symmetric solutions of the Navier-Stokes equations for the isentropic compressible fluids. Math. Methods Appl. Sci. 28, 1-28 (2005)

14. Ding, S, Wen, H, Yao, L, Zhu, C: Global classical spherically symmetric solution of compressible isentropic Navier-Stokes equations with vacuum. SIAM J. Math. Anal. 44, 1257-1278 (2012)

15. Huang, X, Li, J, Xin, Z: Global well-posedness of classical solutions with large oscillations and vacuum to the three-dimensional isentropic compressible Navier-Stokes equations. Commun. Pure Appl. Math. 65, 549-585 (2012)

16. Li, J, Liang, Z: Local well-posedness of strong and classical solutions to Cauchy problem of the two-dimensional barotropic compressible Navier-Stokes equations with vacuum. J. Math. Pures Appl. 102, 640-671 (2014)

17. Li, J, Xin, Z: Global well-posedness and large time asymptotic behavior of classical solutions to the compressible Navier-Stokes equations with vacuum. arXiv:1310.1673v

18. Xin, Z: Blowup of smooth solutions to the compressible Navier-Stokes equation with compact density. Commun. Pure Appl. Math. 51, 229-240 (1998)

19. Jiu, Q, Li, M, Ye, Y: Global classical solution of the Cauchy problem to $1 \mathrm{D}$ compressible Navier-Stokes equations with large initial data. J. Differ. Equ. 257(2), 311-350 (2014) 